\title{
Сорбция гистидина ионообменниками различной природы
}

\author{
(C) 2020 Хохлова О.Н., Фролова К.Э. \\ Воронежский государственный университет, Воронеж \\ Поступила в редакцию 14.11.2020 г.
}

DOI: $10.17308 /$ sorpchrom.2020.20/3140

С целью сравнения сорбции полифункциональных веществ на ионообменниках различной природы исследовано поглощение гистидина гидрохлорида из водных растворов анионообменником АН-251 в хлоридной ионной форме, катионообменником КБ-4П-2 в натриевой ионной форме и полиам-

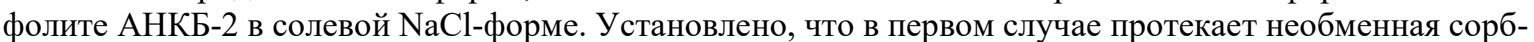
ция за счет ион-дипольных взаимодействий между сорбционным центром «функциональная группа противоион» и заряженной группой аминокислоты с участием молекул воды. На катионообменнике и полиамфолите протекает ионный обмен, поскольку в системе присутствует два сорта катионов $\mathrm{Na}^{+}$и $\mathrm{His}^{+}$, величины которого, однако, невелики. В этих же системах протекает необменное поглощение за счет ион-дипольных взаимодействий противоположно заряженных структурных элементов с участием молекул расторителя, которые, могут протекать с противоионом любой природы, возникающим в системе за счет ионного обмена.

Установлено, что независимо от природы сорбента количество поглощенного гистидина приблизительно одинаково из-за дополнительных взаимодействий, протекающих в системе с участием сорбционных центров (в КБ-4П-2 и АНКБ-2) и малого набухания ионита (АН-251).

Проведено термодинамическое описание исследуемых систем с использованием подходов, основанных на представлении о стехиометричности закрепления вещества в сорбенте. Рассчитаны величины коэффициентов равновесия, исправленных коэффициентов равновесия, коэффициентов активности компонентов фазы сорбента, и термодинамических констант ионообменного и необменного равновесия, а также дифференциальные энергии Гиббса, относящиеся к ионообменнику определенного состава. В качестве состояния сравнения для обоих случаев выбирается состояние равновесия ионообменника в моноионной форме с водой.

Установлено, что в процессе сорбции отклонение систем от состояния сравнения незначительное, что связано с малым количеством поглощенного вещества по сравнению с количеством сорбционных центров, термодинамические контанты равновесия близки к единице. Совместное протекание ионного обмена и необменной сорбции оказывает большее влияние на состояние сорбента по сравнению с необменной сорбцией в силу изменения ионной формы сорбента с минеральной на органическую.

Ключевые слова: гистидин, катионообменник, анионообменник, полиамфолит, ион-дипольные взаимодействия, термодинамическое описание.

\section{Введение}

Поскольку аминокислоты имеют два типа функциональных групп - кислотные $(-\mathrm{COOH})$ и основные $\left(-\mathrm{NH}_{2}\right)$, то ион-дипольные взаимодействия в растворах и сорбентах могут происходить по каждой из них. Для аминокислот, имеющих ионогенные группы в боковом радикале, количество сочетаний в такого типа взаимодействиях значительно выше. Различие в строении аминокислот обуславливает их особенности поглощения сорбентами. Среди ароматических цвиттерлитов с этой точки зрения 
наиболее интересен гистидин, имеющий в боковом радикале две функциональные группы.

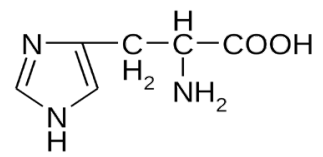

Для реализации всех возможных вариантов взаимодействий в сорбенте необходимо наличие сорбционных центров обоих типов - кислотной и основной природы. Для сравнения исследованы слабокислотный катинообменник КБ-4П-2, низкоосновный анионообменник АН-251 и полиамфолит АНКБ-2, имеющий те же функциональные группы - карбоксильную и азот в бензольном кольце. Целью работы являлось выявление особенностей сорбции и термодинамическое описание поглощения гистидина ионообменниками различной природы в солевой ионной форме.

\section{Экспериментальная часть}

Сорбцию гистидина гидрохлорида проводили в статических условиях из водных растворов. Для исключения возможности перезарядки сорбата в фазе сорбента последние использовали в солевой форме $-\mathrm{Na}^{+}, \mathrm{Cl}^{-}$и $\mathrm{Na}^{+} \mathrm{Cl}^{-}$для катионо-, анионо- и биполярного ионообменника соответственно. Анализ равновесных растворов на содержание аминокислоты проводили спектрофотометрически при 211 нм, содержание противоионов натрия определяли с помощью атомно-эмиссионной спектроскопии, хлора - титриметрически. Суммарное содержание гистидина в сорбенте находили по разнице концентраций до и после сорбции с учетом объема и массы контактирующих фаз, количество ионообменно поглощенной аминокислоты определяли по количеству вытесненного противоиона $\left(\mathrm{Na}^{+}\right)$.

\section{Обсуждение результатов}

Водный раствор гистидина гидрохлорида имеет $\mathrm{pH} 3.9$, что обусловливает существование в растворе однозарядного катиона этой аминокислоты [1]. Сорбция гистидина на анионообменнике протекает без вытеснения противоиона, т.е. необменно, т.к. аминокислота выступает в виде коиона. Закрепление сорбата в фазе сорбента исходит за счет ион-дипольного взаимодействия между противоионом $\mathrm{Cl}^{-}$и положительно зараженной аминогруппой гистидина с участием молекул воды, аналогично изложенному в $[2,3]$.

На катионообменнике и биполярном сорбенте возможен и ионный обмен, и необменное поглощение, т.к. в системе присутствует два сорта ионов $\mathrm{Na}^{+}$и $\mathrm{His}^{+}$. Подтверждение этому получено экспериментально при анализе равновесных растворов на содержание сорбируемой аминокислоты и вытесняемого противоиона $\mathrm{Na}^{+}$. При этом ион-дипольные взаимодействия, обуславливающие необменное поглощение, возможны как с исходным минеральным противоионом, так и ионоообменно поглощенным гистидином за счет притяжения противоположно заряженных структурных элементов с участием молекул воды. Кроме того, на всех сорбентах возможны гидрофобные взаимодействия радикала ароматической аминокислоты с матрицей, однако они слабо выражены у наиболее гидрофильного из ароматических аминокислот гистидина[4].

На рисунках 1 и 2. представлены изотермы сорбции аминокислоты на исследуемых сорбентах. 


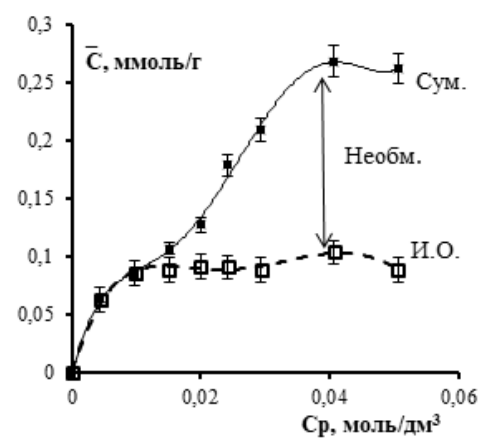

a

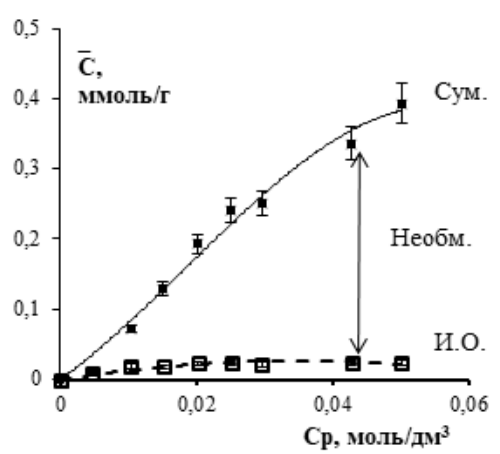

6

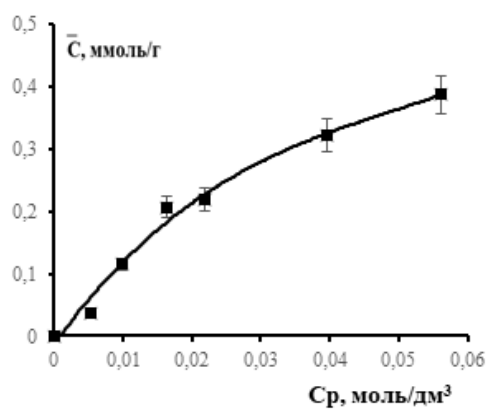

B

Рис. 1. Изотермы сорбции гистидина на ионообменниках а - КБ-4П-2 $\left(\mathrm{Na}^{+}\right)$, б - АНКБ-2 $(\mathrm{NaCl})$, в - АН-251( $\left.\mathrm{Cl}^{-}\right)$

Fig. 1. Sorption isotherms of histidine on ion exchangers a $-\mathrm{KB}-4 \mathrm{P}-2\left(\mathrm{Na}^{+}\right)$, $\mathrm{b}-\mathrm{ANKB}-2(\mathrm{NaCl}), \mathrm{c}-\mathrm{AN}-251\left(\mathrm{Cl}^{-}\right)$

При рассмотрении влияния природы сорбентов на величину поглощения аминокислоты необходимо отметить, что независимо от природы их функциональных групп, емкости и содержания воды, сорбция гистидина мала и составляет не более 10 $\%$ от общего количества сорбционных центров. Это, вероятно, связано в первую очередь с тем, что фиксированный электролит функциональная группа-противоион является не только сорбционным центром, но препятствует поглощению веществ ионообменниками. В рассматриваемых системах, он находится в солевой форме и является полностью диссоциированным, а его внутренний раствор в силу малого содержания воды, является концентрированным. Это приводит к препятствию поглощения как электролитов, так и неэлектролитов, в первом случае в силу Доннановсконо вытеснения, а во втором - в силу высаливания из сорбента [5].

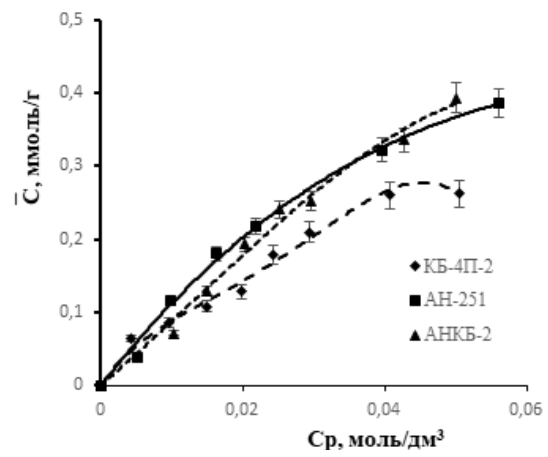

Рис. 2. Изотермы необменной сорбции гистидина на ионообменниках КБ-4П-2 $\left(\mathrm{Na}^{+}\right)$, АН-251 $\left(\mathrm{Cl}^{-}\right)$и АНКБ-2 $(\mathrm{NaCl})$

Fig. 2. Isotherms of non-exchangeable sorption of histidine on ion exchangers $\mathrm{KB}-4 \mathrm{P}-2\left(\mathrm{Na}^{+}\right), \mathrm{AN}-251\left(\mathrm{Cl}^{-}\right)$, and ANKB-2 $(\mathrm{NaCl})$ 
Не смотря на особенности каждого сорбента, суммарные величины поглощения гистидина приблизительно одинаковы. Так КБ-4П-2 имеет наибольшую емкость, и поглощение по двум механизмам, однако это не приводит к большим значениям общей сорбции аминокислоты, вероятно, из-за протонирования части карбоксильных групп сорбента при применении гидрохлорида гистидина. АНКБ-2 имеет оба типа групп (катионообменные и анионообменные), что должно приводить к значительной сорбции гистидина за счет реализации всех возможных типов ион-дипольных взаимодействий и ионного обмена, что, однако, не происходит из-за того, что значительная часть кислотных и основных групп полиамфолита взаимодействуют между собой [6], что препятствует образованию связи с сорбатом. Анионообменник АН-251 хуже всех набухает, что, вероятно, является основной причиной малых величин поглощения аминокислоты на этом сорбенте.

Для термодинамического описания необменной сорбции веществ ионообменниками можно использовать подход, в основу которого положены представления о стехиометричности необменного поглощения:

$$
\beta A+\bar{R} \leftrightarrow \overline{R \mathrm{~A}_{\beta}},
$$

где $\mathrm{A}$ - сорбируемое вещество, $\mathrm{R}$ - свободные сорбционные центры функциональная группа-противоион, $\overline{R A_{\beta}}$ - сорбционные центры, связанные с поглощенным веществом, $\beta$ - коэффициент стехиометрии. Правомочность такого представления процесса сорбции обусловлена схемами взаимодействия, представленными в [7]. При этом сорбционными центрами сорбента являются все функциональные группы в фазе ионообменника.

Равновесие (1) характеризуется константой (2)

$$
K_{A}=\frac{\left(\bar{c}_{R A_{\beta A}} \bar{\gamma}_{R A_{\beta A}}\right)}{\left(c_{A} \gamma_{A}\right)^{\beta_{A}} \bar{c}_{R} \bar{\gamma}_{R}}=\widetilde{\mathrm{K}}_{A} \frac{\bar{\gamma}_{R A_{\beta A}}}{\bar{\gamma}_{R}},
$$

где $\mathrm{C}_{\mathrm{A}}$ и $\gamma_{\mathrm{A}}$ - концентрация и коэффициент активности поглощаемого вещества в растворе, $\bar{C}_{\mathrm{RA}}, \overline{\mathrm{C}}_{\mathrm{R}}, \bar{\gamma}_{\mathrm{RA}}, \bar{\gamma}_{\mathrm{R}}-$ концентрации и коэффициенты активности связанных с сорбатом и свободных сорбционных центров в фазе сорбента; $\mathrm{K}_{\mathrm{A}}, \widetilde{\mathrm{K}}_{A}$ - термодинамическая константа и коэффициент равновесия сорбции.

При параллельном протекании ионного обмена и необменной поглощения оба процесса представляют стехиометрическими уравнениями $[8,9]$.

Так ионный обмен записывается как

$$
z_{2} I_{1}+z_{1} \bar{I}_{2} \Leftrightarrow z_{2} \bar{I}_{1}+z_{1} I_{2}
$$

где $\mathrm{I}_{1}$ - исходная ионная форма ионообменника, $\mathrm{I}_{2}$ - входящий органический ион, $\mathrm{Z}_{1}$, $\mathrm{z}_{2}$ - заряды обменивающихся ионов.

Необменная сорбция, которая протекает совместно с ионным обменом, трактуется как сверхэквивалентная, и может быть записана как

$$
\beta I_{1}+\bar{I}_{1} \Leftrightarrow \bar{I}_{3},
$$

где $\overline{\mathrm{I}}_{3}$ - образующийся ассоциат, следующего состава: $\bar{I}_{3} \equiv \overline{I_{1}\left(I_{1}\right)}, \beta$ - стехиометрический коэффициент необменной сорбции.

Константы равновесия ионного обмена и необменного поглощения записываются соответственно как

$$
\begin{aligned}
& K_{1}=\frac{\bar{a}_{1}^{z_{2}} \cdot a_{2}^{z_{1}}}{\bar{a}_{2}^{z_{1}} \cdot a_{1}^{z_{2}}}=\bar{K}_{1} \frac{\bar{\gamma}_{1}^{z_{2}}}{\bar{\gamma}_{2}^{z_{1}},} \\
& K_{2}=\frac{\bar{c}_{3} \bar{\gamma}_{3}}{\left(c_{1} \gamma_{1}\right)^{\beta} \overline{c_{1}} \bar{\gamma}_{1}}=\bar{K}_{2} \frac{\bar{\gamma}_{3}}{\bar{\gamma}_{1}},
\end{aligned}
$$


где $a, C, \gamma, z$ - активность, концентрация, коэффициент активности и заряд соответствующих компонентов в фазах раствора, в случае сорбента величины отмечаются сверху чертой. $\widetilde{K}-$ исправленный коэффициент равновесия соответствующего процесса.

Оба подхода предусматривают нахождение из экспериментальных данных коэффициентов равновесия сорбционных процессов, затем расчет исправленных коэффициентов равновесия с учетом коэффициентов активности ионов в растворе, затем нахождение коэффициентов активности компонентов фазы сорбента, с помощью которых находят термодинамические константы ионообменного и необменного равновесия. В качестве состояния сравнения для обоих случаев выбирается состояние равновесия ионообменника в моноионной форме с водой [7-9]. Необходимо отметить, что вклад ионного обмена в суммарную сорбцию гистидина полиафолитом АНКБ-2 мал, поэтому описание этой системы проводили, используя первый подход.

На рисунке 3 представлено изменение исправленных коэффициентов равновесия в исследуемых системах. Для необменной сорбции эти величины выше, чем для ионного обмена на катионообменнике, проходят через максимум и стремятся к постоянным значениям при больших концентрациях. Ионный обмен характеризуется малыми значениями $\ln \widetilde{\kappa}$ в силу того, что емкость катионообменника велика, а количество поглощенной аминокислоты мало, и большая часть сорбционных центров остается в исходной ионной форме. Падение этих величин, вероятно, связано с конкурирующим процессом протонирования функциональных групп сорбента ионами водорода гидрохлорида гистидина.

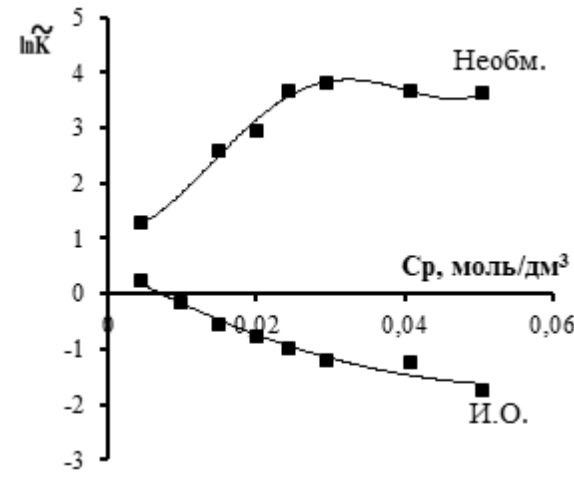

a

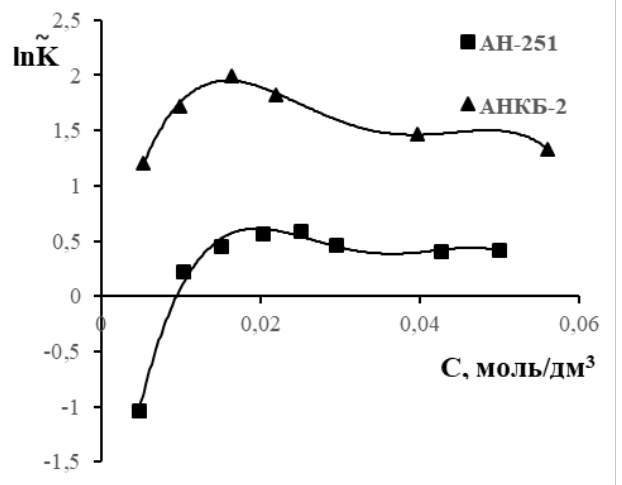

б

Рис. 3. Зависимость логарифмов коэффициента равновесия сорбции гистидина от равновесной концентрации раствора на ионообменниках:

$$
\text { a - КБ-4П-2( } \left.\left.\mathrm{Na}^{+}\right), \text {б - АН-251 }\left(\mathrm{Cl}^{-}\right) \text {и АНКБ-2( } \mathrm{NaCl}\right)
$$

Fig. 3. Dependence of the logarithms of the equilibrium coefficient of the sorption of histidine on the equilibrium concentration of the solution on ion exchangers:

$$
\mathrm{a}-\mathrm{KB}-4 \mathrm{P}-2\left(\mathrm{Na}^{+}\right), \mathrm{AN}-251\left(\mathrm{Cl}^{-}\right), \mathrm{b}-\mathrm{ANKB}-2(\mathrm{NaCl})
$$

На рисунке 4 представлено изменение коэффициентов активности компонентов фазы сорбента в исследуемых системах, рассчитанных согласно [7-9]. Полученные коэффициенты активности свободных сорбционных центров $\overline{\gamma_{\mathrm{R}}}$ во всех исследуемых системах практически не отличаются от единицы, а коэффициенты активности

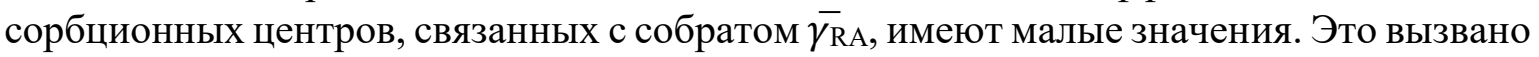
тем, что количество поглощенного вещества мало по сравнению с количеством сорбционных центров, следовательно, состояние системы незначительно отклоняется от отсчетного состояния. Исключение составляет система с участием катионообменника, в которой наблюдается большие величины коэффициентов активности ионообменно 
поглощенного вещества при наименьшем отклонении системы от состояния сравнения.

Необходимо отметить, что при детальном рассмотрении изменения коэффициентов активности свободных сорбционных центров в случае ионного обмена это изменение выражено ярче, т.к. в системе происходит смена минерального противоиона на органический.

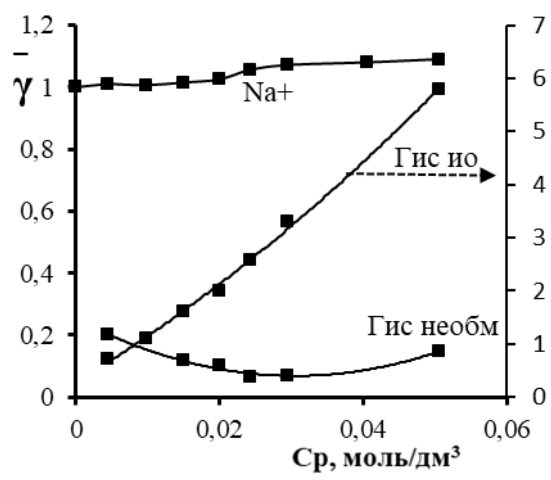

a

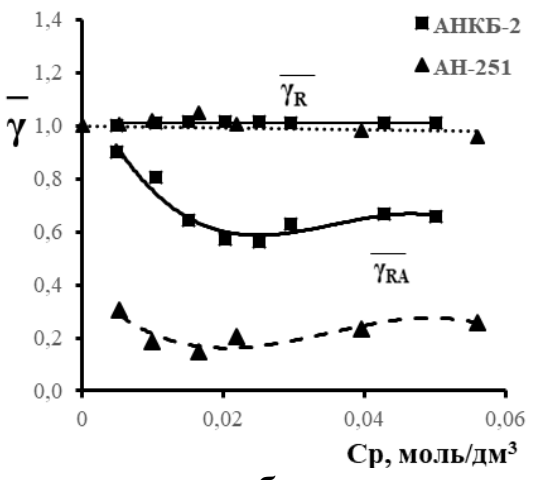

6

Рис. 4. Зависимости коэффициентов активности свободных сорбционных центров $\left(\gamma_{\mathrm{R}}\right)$ и связанных с гистидином $\left(\gamma_{\mathrm{RA}}\right)$ в фазе сорбента от равновесной концентрации раствора аминокислоты на ионообменниках: a - КБ-4П-2 $\left(\mathrm{Na}^{+}\right)$, б - АН-251 $\left(\mathrm{Cl}^{-}\right)$и АНКБ-2 $(\mathrm{NaCl})$

Fig. 4. Dependences of the activity coefficients of free sorption centres $(\bar{R})$ and centres bound with histidine (RA) in the sorbent phase on the equilibrium concentration of the amino acid solution on ion exchangers: $\mathrm{a}-\mathrm{KB}-4 \mathrm{P}-2\left(\mathrm{Na}^{+}\right), \mathrm{b}-\mathrm{AN}-251\left(\mathrm{Cl}^{-}\right)$ and ANKB-2 $(\mathrm{NaCl})$

Для описания сорбционных равновесий на различных стадиях процесса информативным является изменение дифференциальных энергий Гиббса, относящихся к ионообменнику определенного состава. Значения $\Delta \mathrm{G}_{\mathrm{n}}$, рассчитывают из величин активности сорбируемых (и десорбируемых в случае ионного обмена) компонентов равновесного раствора [7-9]. Во всех исследуемых системах величины дифференциальных энергий Гиббса имеют отрицательные значения, что свидетельствует о самопроизвольности и энергетической выгодности процессов. Необходимо отметить, что по мере насыщения сорбента происходит уменьшение величин $\Delta \mathrm{G}_{\mathrm{n}}$ в одном диапазоне значений, однако для ионного обмена эти величины изменяются в более узком концентрационном интервале, что соответствует малой степени заполнения сорбента по этому механизму. Интересно отметить, что в области концентраций гистидина в катионообменнике 0.09 ммоль/Г дифференциальные энергии Гиббса обоих процессов совпадают.

Для исследуемых систем с учетом коэффициентов активности компонентов фазы сорбента получены значения термодинамических констант равновесия. Рассчитанные константы равновесия необменной сорбции близки к единице для всех систем, а для ионного обмена составляют 0.92 , что определяется малым количеством поглощенного вещества по обоим механизмам и близости изотерм к линейному виду. 


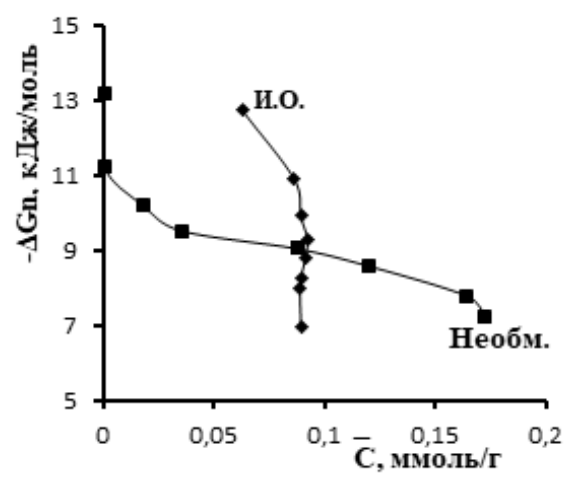

a

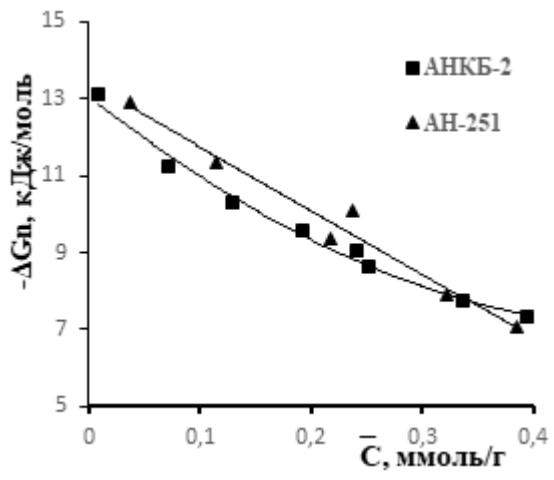

6

Рис. 5. Зависимость дифференциальной энергии Гиббса от содержания гистидина в фазе сорбента на ионообменниках: $\mathrm{a}-$ КБ-4П-2 $\left(\mathrm{Na}^{+}\right)$, б - АН-251( $\left.\mathrm{Cl}^{-}\right)$и АНКБ-2 $(\mathrm{NaCl})$

Fig. 5. Dependence of differential Gibbs energy on the content of histidine in the sorbent phase on ion exchangers: a-KB-4P-2 $\left(\mathrm{Na}^{+}\right)$, $\mathrm{b}-\mathrm{AN}-251\left(\mathrm{Cl}^{-}\right)$and ANKB-2 $(\mathrm{NaCl})$

\section{Заключение}

Таким образом, необменная сорбция гистидина гидрохлорида на ионообменниках различной природы катионообменнике КБ-4П-2( $\left.\mathrm{Na}^{+}\right)$, анионообменнике $\mathrm{AH}-251\left(\mathrm{Cl}^{-}\right)$и полиамфолите АНКБ-2 в $\mathrm{NaCl}$-форме имеет близкие термодинамические характеристики, в силу действия одинаковых сил закрепления в фазе сорбента и малого количества поглощенного вещества. Совместное протекание ионного обмена и необменной сорбции в системе КБ-4П-2 (Na) + His оказывает большее влияние на состояние сорбента по сравнению с необменной сорбцией в силу изменения ионной формы сорбента с минеральной на органическую.

\section{Список литературы}

1. Селеменев В.Ф., Хохлов В.Ю., Бобрешова О.В. Физико-химические основы сорбционных и мембранных методов выделения и разделения аминокислот. Воронеж. Изд-во Воронеж. ун-та. 2001. 299 с.

2.Трунаева Е.С., Хохлова О.Н., Хохлов В.Ю. // Сорбционные и хроматографические проиессы. 2018. Т. 18 № 2. С. 142-149.

3. Трунаева Е.С., Хохлова О.Н., Хохлов В.Ю. // Журнал структурной химии. 2017. Т. 58. № 1. C. 23-28 .

4. Демин А.А., Чернова И.А., Шатаева Л.К. Ионообменная сорбция биологически активных веществ. СПб. Изд-во С.-Петерб. ун-та. 2008. $154 \mathrm{c}$.
5. Гельферих Ф. Иониты: Основы ионного обмена. М. Изд-во иностр. лит-ры. 1962. 490 c.

6. Углянская В.А., Чикин Г.А., Селеменев В.Ф. Инфракрасная спектроскопия ионообменных материалов. Воронеж. Изд-во ВГУ. 1989. $208 \mathrm{c}$.

7. Трунаева Е.С., Хохлова О.Н., Хохлов В.Ю. // Журнал физической химии. 2018. Т. 92. № 12. С. 1920-1924 .

8. Хохлова О.Н. // Журнал физической химии. 2014. Т. 88. № 7-8. С. 1243-1246.

9. Хохлова О.Н. // Журнал физической химии. 2014. Т. 88. № 7-8. С. 1247-1250. 


\title{
Sorption of histidine by different types ion exchangers
}

\author{
(C) 2020 Khokhlova O.N., Frolova K.E.
}

Voronezh State University, Voronezh

In order to compare the sorption of polyfunctional substances on ion exchangers of different nature, the absorption of histidine hydrochloride from aqueous solutions by an AN-251 anion exchanger in chloride ionic form, KB-4P-2 cation exchanger in sodium ionic form, and ANKB-2 polyampholite in $\mathrm{NaCl}$ salt form was studied. It was established that in the first case, non-exchange sorption occurs due to ion-dipole interactions between the sorption centre "functional group counterion" and the charged group of an amino acid with the participation of water molecules. Ion exchange occurs on the cation exchanger and polyampholite, since the system contains two types of cations $\mathrm{Na}^{+}$and $\mathrm{His}^{+}$, the values of which, however, are small. In these systems, non-exchange absorption occurs due to ion-dipole interactions of oppositely charged structural elements with the participation of solvent molecules, which can occur with a counterion of any nature arising in the system due to ion exchange.

It was found that regardless of the nature of the sorbent, the amount of absorbed histidine is approximately the same due to additional interactions occurring in the system with the involvement of sorption centres (in KB-4P-2 and ANKB-2) and a small swelling of ion exchanger (AN-251).

The thermodynamic description of the systems using approaches based on the idea of stoichiometry of substance fixation in the sorbent was carried out. The values of the equilibrium coefficients, corrected equilibrium coefficients, activity coefficients of the sorbent phase components, and thermodynamic constants of ion-exchange and non-exchange equilibrium, as well as Gibbs differential energies related to an ion exchanger of a certain composition, were calculated. The state of equilibrium of the ion exchanger in the monoionic form with water was chosen as the comparison state for both cases.

The deviation of systems from the comparison state during sorption was insignificant due to the small amount of absorbed compound in comparison with the number of sorption centres. The thermodynamic equilibrium constants were close to one. The joint ion exchange and non-exchange sorption had a greater influence on the state of the sorbent compared to non-exchange sorption due to the change in the ionic form of the sorbent from mineral to organic.

Keywords: histidine, cation exchanger, anion exchanger, polyampholyte, ion-dipole interactions, thermodynamic description.

\section{References}

1. Selemenev V.F., Khokhlov V.Yu., Bobreshova O.V. Fiziko-khimicheskie osnovy sorbtsionnykh i membrannykh metodov vydeleniya i razdeleniya aminokislot. Voronezh, Izd-vo Voronezh. yn-ta, 2001, 299 p.

2.Trynaeva E.S., Khokhlova O.N., Khokhlov V.Yu., Sorbtsionnye $i$ khromatograficheskie protsessy, 2018, Vol. 18, No 2, pp. 142-149.

3. Trynaeva E.S., Khokhlova O.N., Khokhlov V.Yu., Zhurnal stryktyrnoy khimii, 2017, Vol. 58, No 1, pp. 23-28.

4. Demin A.A., Chernova I.A., Shataeva L.K. Ionoobmennaya sorbtsiya biologicheski aktivnykh veщestv. SPb, Izd-vo S.-Peterb. yn-ta, 2008, $154 \mathrm{p}$.

Хохлова Оксана Николаевна - к.Х.н., доцент кафедры аналитической химии, химический факультет, Воронежский Государственный Университет, Воронеж

Фролова Ксения Эдуардовна - студент кафедры аналитической химии, химический факультет, Воронежский Государственный Университет, Воронеж
5. Gelferikh F. Ionity: Osnovy ionnogo obmena. M, Izd-vo inostr. lit-ry, 1962, 490 p.

6. Yglyanskaya V.A., Chikin G.A., Selemenev V.F. Infrakrasnaya spektroskopiya ionoobmennykh materialov. Voronezh, Izd-vo VGU, 1989, $208 \mathrm{p}$.

7. Trynaeva E.S., Khokhlova O.N., Khokhlov V.Yu., Zhurnal fizicheskoy khimii, 2018, Vol. 92, No 12, pp. 1920-1924.

8. Khokhlova O.N., Zhurnal fizicheskoy khimii, 2014, Vol. 88, No 7-8, pp. 1243-1246.

9. Khokhlova O.N., Zhurnal fizicheskoy khimii, 2014, Vol. 88, No 7-8, pp. 1247-1250.

Khokhlova Oksana N. - associate professor, department of analytical chemistry, chemical faculty, Voronezh State University, Voronezh, e-mail: okxox@yandex.ru

Frolova Ksenya E. - student, department of analytical chemistry, chemical faculty, Voronezh State University, Voronezh, e-mail: frolova.k@icloud.com 